Check for updates

Cite this: RSC Adv., 2018, 8, 9243

Received 29th November 2017

Accepted 23rd February 2018

DOI: $10.1039 / c 7 r a 11153 c$

rsc.li/rsc-advances

\section{Preliminary characterization of the structure and immunostimulatory and anti-aging properties of the polysaccharide fraction of Haematococcus pluvialis $\uparrow$}

\begin{abstract}
Xiaojuan Liu, (D)*ab Miao Zhang, ${ }^{\text {ab }}$ Han Liu, $^{\text {ab }}$ Aimei Zhou, ${ }^{\text {ab }}$ Yong Cao ${ }^{\text {ab }}$ and Xin Liu ${ }^{\text {ab }}$
In this study, a polysaccharide fraction (HPP-c3-s1) was obtained from Haematococcus pluvialis using DEAE-52 anion exchange and Sephacryl S400 chromatographies. The structure of HPP-c3-s1 was partially characterized and its biological activity was investigated. HPP-c3-s1 is a homogeneous polysaccharide with a molecular weight of $23413 \mathrm{kDa}$ as determined by high-performance gel permeation chromatography and polyacrylamide gel electrophoresis. Periodate oxidation and Smith degradation analysis combined with GC-MS determined that HPP-c3-s1 contains $1 \rightarrow 2,1 \rightarrow 3,1 \rightarrow 4$, and probably a few $1 \rightarrow 6$ glycosyl linkages. Fourier transform infrared spectroscopy and nuclear magnetic resonance analyses indicate HPP-c3-s1 is a pyranose containing an amino and O-acetyl group. The sugar chains in HPP-c3-s1 contain both $\alpha$ and $\beta$ glycosidic configurations, where the $\beta$ configuration is the primary form. It was observed that the HPP-c3-s1 nanostructure had linear and branched forms at a concentration of $5 \mu \mathrm{g} \mathrm{mL}^{-1}$ and created different sized aggregates at a higher concentration of $50 \mu \mathrm{g}$ $\mathrm{mL}^{-1}$. Furthermore, HPP-c3-s1 had significant immunostimulatory effects on splenocytes and $B$ lymphocytes. In assays assessing anti-aging effects, HPP-c3-s1 extended the mean survival of Caenorhabditis elegans without adversely affecting reproduction. In addition, treatment with HPP-c3-s1 resulted in delays in age-related physiologic parameters, including body movement, rates of head swing and body bending, and accumulation of intestinal lipofuscinosis in C. elegans. Overall, these results suggest HPP-c3-s1 has remarkable immunomodulatory and anti-aging properties that may serve as the basis for development of functional foods and dietary supplements.
\end{abstract}

\section{Introduction}

In recent years, algal polysaccharides have been the focus of interest as novel bioactive materials, primarily due to their novel structures and potential biological functions. ${ }^{1}$ Sulfated polysaccharides contain sulfated ester groups within their sugar residues, which are common components of a wide range of macroalgae (seaweeds). ${ }^{2}$ The chemical structures vary based on the algal species and extraction procedure. Substantial studies have found these seaweed polysaccharides possess unique anticoagulant, ${ }^{3}$ anti-inflammatory, ${ }^{4}$ antitumor, ${ }^{5}$ antioxidant, ${ }^{6}$ and immunomodulating properties, ${ }^{7}$ suggesting potential for use in pharmaceutical applications. Therefore, polysaccharides originating from red and brown macroalgae have attracted

\footnotetext{
${ }^{a}$ Department of Food Science, College of Food Science, South China Agricultural University, Guangzhou 510642, China. E-mail: liuxj@scau.edu.cn; Tel: +86-20-85286234

${ }^{b}$ Research Center of Natural Active Compound Engineering Technology of Guangdong Province, Guangzhou 510642, China

$\dagger$ This manuscript has been thoroughly edited by a native English speaker from an editing company.
}

much attention as functional food additives and natural therapeutic agents in the pharmaceutical field.

Microalgae, which are distinct from macroalgae, are photosynthetic unicellular alga that utilize inorganic nutrients present in marine and fresh water, are easily cultivated, and have been investigated as foods for human consumption and attractive sources of functional food. ${ }^{8}$ Microalgae contain an abundance of polysaccharides, some of which may possess diverse bioactivity with pharmacological significance. ${ }^{9}$ However, fewer studies have been conducted on microalgal polysaccharides than on macroalgal polysaccharides. For example, sulfated HCP from the green microalgae Haematococcus lacustris has potent immunomodulating activity, ${ }^{\mathbf{1 0}}$ while sulfated EPS from the microalgae Gyrodinium impudicum strain KG03 has antiviral activity. ${ }^{11}$

Haematococcus pluvialis is a unicellular green alga found in both marine and fresh water and has garnered much attention, including in the form of studies on producing and bioengineering astaxanthin, an excellent antioxidant that can be isolated from $H$. pluvialis. $^{12}$ However, few studies have been conducted on the chemical structures and potential bioactivity 
of polysaccharides in $H$. pluvialis, although studies have determined $H$. pluvialis polysaccharides have antioxidant and thrombolytic properties. ${ }^{13}$ Accordingly, additional searching for active polysaccharides in $H$. pluvialis aids in the development of new functional foods. For these reasons, a water-soluble polysaccharide, HPP-c3-s1, was isolated and purified from $\mathrm{H}$. pluvialis. In the present study, the chemical structure of HPP-c3-s1 was characterized, and its immunomodulatory and anti-aging properties were assessed using several in vitro and in vivo tests.

\section{Materials and methods}

\subsection{Materials}

Dried Haematococcus pluvialis residue was obtained from Jingzhou Natural Astaxanthin Inc. Male BALB/c mice (6 to 8 weeks old) were purchased from the SPF Laboratory Animal Center at Southern Medical University. All animal studies were approved by South China Agricultural University Institutional Animal Care and Use Committee and performed in strict accordance of NIH guidelines for animal care. Reagents used in cell-based assays included RPMI 1640 medium, fetal bovine serum (FBS), and $100 \times$ penicillin and streptomycin purchased from Gibco (Grand Island, NY, USA), and dimethyl sulphoxide (DMSO), 3-(4,5-dimethylthiazol-2-yl)-2,5-diphenyltetrazolium bromide (MTT), concanavalin A (ConA), lipopolysaccharide (LPS), standard sugars, and dextran standard purchased from Sigma-Aldrich Co., Ltd (St. Louis, MO, USA). Caenorhabditis elegans wild type N2 (var. Bristol) were obtained from the Caenorhabditis Genetics Center (CGC, University of Minnesota, Minneapolis, MN, USA). The Escherichia coli OP50 uracil mutant was provided by the College of Resource and Environment at the South China Agricultural University. All other chemical reagents used were analytical grade.

\subsection{Preparation of polysaccharide HPP-c3-s1}

Crude polysaccharide (HPP) was extracted from $H$. pluvialis residues using ultrasonic-assisted extraction, anhydrous ethanol $(1: 4, \mathrm{v} / \mathrm{v})$ precipitation, and then deproteination with Sevag reagent ( $n$-butanol: chloroform $=1: 5, \mathrm{v} / \mathrm{v}$ ) as described in a previous report. ${ }^{14}$ The HPP was purified using a DEAEcellulose-52 chromatography column $(3.0 \times 35 \mathrm{~cm})$ and then eluted stepwise with distilled water and a gradient of $0.1,0.3$,

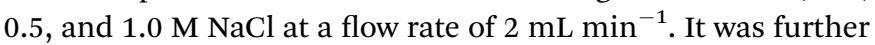
purified by Sephacryl S-400 $(1.6 \times 70 \mathrm{~cm})$ gel permeation chromatography, where it was eluted with distilled water at a flow rate of $0.3 \mathrm{~mL} \mathrm{~min}^{-1}$. One of the obtained purified polysaccharide fractions was designated HPP-c3-s1.

\subsection{Determination of homogeneity and molecular weight}

The homogeneity and molecular weight of HPP-c3-s1 were measured using high-performance gel permeation chromatography (HPGPC) on a LC-10A HPLC system (Shimadzu, Japan) with a RID-10A detector. ${ }^{15}$ A gel-filtration chromatographic column system comprised of TSK-Gel G5000 PWXL and TSK-Gel G3000 PWXL columns (both $7.8 \mathrm{~mm} \times 30 \mathrm{~cm}$, Tosoh, Japan) connected in series were maintained at $40{ }^{\circ} \mathrm{C}$ and an injection volume of $20 \mu \mathrm{L}$ was used. Ultra-pure water was used as the mobile phase at a flow rate of $0.6 \mathrm{~mL} \mathrm{~min}^{-1}$. Dextran T-series standards of varying molecular weights (T-5000, T-12000, T25000, T-50000, T-80000, T-150000, T-270000, T-410000, and $\mathrm{T}-670000)$ were prepared to estimate the molecular weight (MW) of the polysaccharide. The equation for the calibration curve of dextran was: $Y=0.2256 X^{2}-5.1357 X+33.715\left(R^{2}=0.9887\right) . X$ : Logarithm of relative molecular mass of dextran; $Y$ : elution volume.

The homogeneity of HPP-c3-s1 was also measured by polyacrylamide gel electrophoresis (PAGE) with 8\% separating and $3.5 \%$ stacking gels. HPP-c3-s1 in boric acid-potassium chloride buffer $\left(0.1 \mathrm{~mL}, 10 \mathrm{mg} \mathrm{mL}^{-1}\right)$ was mixed at a $2: 1$ ratio with sample buffer (boric acid-potassium chloride buffer, pH 9.0 containing $0.2 \%$ bromophenol blue and $40 \%$ glycerol) and centrifuged at $10000 \mathrm{rpm}$ for $5 \mathrm{~min}$ at $4{ }^{\circ} \mathrm{C}$. Sample solution $(10 \mu \mathrm{L})$ was loaded into each lane of the gel. The samples were electrophoresed initially at $40 \mathrm{~V}$ for $20 \mathrm{~min}$ and then at $110 \mathrm{~V}$ for $1 \mathrm{~h}$. The gels were washed with a rinse solution (1:50:50 of glacial acetic acid, ethanol, and distilled water), and then oxidized with periodic acid for $40 \mathrm{~min}$. The gels were then rinsed with sodium metabisulfite solution, incubated with Schiff staining solution for $30 \mathrm{~min}$, and then destained with sodium metabisulfite solution three times for 5 min each. The gels were optically scanned using a Universal Hood II (Bio-rad Company, USA) gel imaging analysis system.

\subsection{Periodate oxidation and smith degradation}

To prepare the monosaccharide derivatives, $10.00 \mathrm{mg}$ hydroxylamine hydrochloride, and $0.5 \mathrm{~mL}$ pyridine were added to $5.00 \mathrm{mg}$ D-ribose, D-glucose, D-mannose, or D-arabinose. The tubes were filled with nitrogen gas and then sealed. The reaction was incubated at $90{ }^{\circ} \mathrm{C}$ for $30 \mathrm{~min}$ and then cooled. Following addition of $0.5 \mathrm{~mL}$ of acetic anhydride, the tubes were once again filled with nitrogen gas and then sealed. The reaction was incubated at $90{ }^{\circ} \mathrm{C}$ for another $30 \mathrm{~min}$, and the saccharide acetate derivative of monosaccharide was obtained. After passing through a $0.22 \mu \mathrm{m}$ filter, $5 \mu \mathrm{L}$ of sample was used for GC-MS analysis.

A solution of $5 \mathrm{mg}$ HPP-c3-s1 mixed with $50 \mathrm{~mL}$ of $15 \mathrm{mM}$ $\mathrm{NaIO}_{4}$ was kept at $4{ }^{\circ} \mathrm{C}$ in the dark. Oxidation was quenched by adding $1.5 \mathrm{~mL}$ ethylene glycol after $24 \mathrm{~h}$. The solution was concentrated, reduced, neutralized, dialyzed, and lyophilized to produce a degraded product. This degraded product $(2 \mathrm{mg}$ dissolved in $2 \mathrm{~mL}$ of water) was further hydrolyzed with $4 \mathrm{M}$ trifluoroacetic acid at $110{ }^{\circ} \mathrm{C}$ for $3 \mathrm{~h}$ in a screw cap tube filled with nitrogen gas. Hydroxylamine hydrochloride $(10 \mathrm{mg})$ and $0.5 \mathrm{~mL}$ of pyridine were added to the hydrolyzed samples and the samples incubated for $30 \mathrm{~min}$ at $90{ }^{\circ} \mathrm{C}$. Next, $0.5 \mathrm{~mL}$ of acetic anhydride was added and the samples were allowed to stand again for $30 \mathrm{~min}$ at $90{ }^{\circ} \mathrm{C} .{ }^{16}$ The resulting alditol acetate product was analyzed by GC-MS. For GC-MS, a DB- 5 column $(0.2 \mathrm{~mm} \times$ $30 \mathrm{~m} \times 0.25 \mu \mathrm{m})$ and high purity helium as a carrier gas were used. The flow rate was $1.2 \mathrm{~mL} \mathrm{~min}^{-1}$, the chromatographic inlet temperature $280{ }^{\circ} \mathrm{C}$, and the column temperature program was set at an initial temperature of $120{ }^{\circ} \mathrm{C}$ for $5 \mathrm{~min}$ followed by 
a temperature rise at $120{ }^{\circ} \mathrm{C}$ at a rate of $20{ }^{\circ} \mathrm{C} \mathrm{min} \mathrm{m}^{-1}$ for $5 \mathrm{~min}$, and then a temperature increase to $280{ }^{\circ} \mathrm{C}$ at a rate of $10{ }^{\circ} \mathrm{C} \mathrm{min}^{-1}$ for $5 \mathrm{~min}$. For mass spectrometry, an EI ion source was used, the electron energy was $70 \mathrm{eV}$, and the transmission line temperature was $280{ }^{\circ} \mathrm{C} .{ }^{17}$

\subsection{Fourier transform infrared spectroscopy (FTIR)}

FTIR spectroscopy was used to analyze the structure of purified HPP-c3-s1. To this end, $1 \mathrm{mg}$ of solid sample was mixed with dry potassium bromide (100 $\mathrm{mg}$ ) and then analyzed on a Nexus FTIR (Nicolet Nexus, Thermo Nicolet Company, USA) with a DTGS KBr detector. The absorptive spectra were recorded in the frequency range of $4000-400 \mathrm{~cm}^{-1} .^{18}$

\subsection{Nuclear magnetic resonance (NMR)}

The ${ }^{1} \mathrm{H}$ NMR spectra of HPP-c3-s1 were recorded using an Avance(C NMR spectrometer (Bruker, Germany) at $600 \mathrm{MHz}$ in $\mathrm{D}_{2} \mathrm{O}$. Freeze-dried sample $(10 \mathrm{mg}$ ) was exchanged three times with $\mathrm{D}_{2} \mathrm{O}$ and spectra were measured in the range of $0-6 \mathrm{ppm} .{ }^{19}$

\subsection{Atomic force microscope (AFM)}

The nanostructure and self-assembly capability of HPP-c3-s1 were determined by AFM. HPP-c3-s1 suspensions were prepared with concentrations of 5 and $50 \mu \mathrm{g} \mathrm{mL}{ }^{-1}$. Exactly $5 \mu \mathrm{L}$ of each sample was dropped onto freshly cleaved mica and air dried overnight. AFM imaging was performed on a MultiMode 8 (Bruker, Germany) instrument at room temperature (approximately $22-25{ }^{\circ} \mathrm{C}$ ) and a relative humidity of $50-60 \%$ on a mica sheet. The tape on the tip was Silicon Tip on Nitride Lever and the cantilever was RTEST ( $\left.T: 650 \mathrm{~nm}, f_{0}: 70 \mathrm{kHz}, k: 0.4 \mathrm{~N} \mathrm{~m}^{-1}\right){ }^{20}$

\subsection{Spleen lymphocyte proliferation assay}

Splenocytes were harvested from mice and then suspended in RPMI-1640 medium. Splenocytes were transferred to a 96-well culture plate at $1.0 \times 10^{6}$ cells per $\mathrm{mL}$ in $200 \mu \mathrm{L}$ complete medium. Each well was treated with $25 \mu \mathrm{L}$ of 3.9, 7.8, 15.6, 31.2, $62.5,125$, or $250 \mu \mathrm{g} \mathrm{mL}{ }^{-1}$ polysaccharide. The plates were incubated at $37{ }^{\circ} \mathrm{C}$ in a humidified $5 \% \mathrm{CO}_{2}$ atmosphere. Cells were treated with $25 \mu \mathrm{L}$ of $10 \mu \mathrm{g} \mathrm{mL}^{-1}$ ConA or $10 \mu \mathrm{g} \mathrm{mL}^{-1} \mathrm{LPS}$ as positive controls. Meanwhile, the blank control was treated with $25 \mu \mathrm{L}$ of the medium. After $44 \mathrm{~h}, 20 \mu \mathrm{L}$ of $5 \mathrm{mg} \mathrm{mL}^{-1}$ MTT was added to each well and the plates were incubated for another $4 \mathrm{~h}$. The plates were centrifuged at $3000 \mathrm{rpm}$ for $10 \mathrm{~min}$, the supernatant was discarded, and then $150 \mu \mathrm{L}$ of DMSO was added to dissolve formazan crystals. The absorbance of the splenocytes in each well was measured at $570 \mathrm{~nm}$ using a microplate reader (ThermoScientific Instruments, USA). ${ }^{21}$

\subsection{Preparation of treatment plates}

HPP-c3-s1 was dissolved in sterile water to final concentrations of 50, 200, and $400 \mu \mathrm{g} \mathrm{mL} \mathrm{m}^{-1}$ according to our pre experiment. Each concentration of HPP-c3-s1 was freshly spotted onto a $60 \mathrm{mM}$ Petri dish containing $10 \mathrm{~mL}$ of nematode growth medium (NGM). All HPP-c3-s1 plates were seeded with E. coli OP50 one day before use. The plates were stored at $4{ }^{\circ} \mathrm{C}$.

\subsection{Lifespan assay}

C. elegans were cultured and maintained at $20{ }^{\circ} \mathrm{C}$ on NGM plates seeded with $E$. coli OP50 as previously described. ${ }^{22}$ All treatments started at the L4 stage after synchronizing with sodium hypochlorite, which kills adult worms but not their eggs. For each treatment, 90 nematodes were transferred to NGM plates previously treated with corresponding test concentrations of HPP-c3-s1 or E. coli OP50 alone as a control. The first day of adulthood was designated day 0 and nematodes were transferred to fresh treatment plates every 2 days to avoid contamination. The survival of the nematodes was noted daily until all the worms had died. Any worms that fled to the wall or cover of the Petri dish and died or drilled into the agar were excluded from the analysis. ${ }^{22}$ Three independent trials were performed per treatment. Data are shown as mean lifespan per trial.

\subsection{Measurement of age-related changes in physiological processes}

Mobility force assay were performed as previously described. ${ }^{23}$ For the egg-laying assay, L4 larvae ( $n=10$ for each treatment) were placed on individual plates with a treatment or control and then transferred to fresh plates every $24 \mathrm{~h}$ until reproduction ceased. The progeny of each nematode were enumerated at the L2 or L3 stage. ${ }^{24}$ The rate of head swing and body bending were determined by counting head swings and sinusoidal motions, respectively. The nematodes were grown on NGM plates containing a treatment or control. Ten nematodes were randomly selected on days 5,10 , and 15 , and the rates of head swings and body bending were recorded at for regular intervals of $30 \mathrm{~s}$ and 1 min, respectively. Each experiment was performed independently in triplicate.

\subsection{Statistical analysis}

Experimental results are expressed as mean \pm SD. Statistically significant differences were determined by one-way ANOVA using SPSS 17.0 (IBM Co., NY, USA). Statistical significance was considered $p<0.05$.

\section{Results and discussion}

\subsection{Homogeneity and molecular weight of HPP-c3-s1}

As shown in Fig. 1A, HPGPC analysis revealed only one single and symmetrical peak for the purified polysaccharide, HPP-c3s1, indicating it was a homogeneous fraction. This peak had a retention time of $12.633 \mathrm{~min}$. According to the calibration curve, $\log M_{\mathrm{w}}=-0.2073 R_{\mathrm{t}}+9.7969$, where $R^{2}=0.995, M_{\mathrm{w}}$ is standard molecular weight, and $R_{\mathrm{t}}$ represents retention time, the average molecular weight of HPP-c3-s1 was calculated to be about $23413 \mathrm{kDa}$. Based on PAGE, HPP-c3-s1 had a single PASpositive band (Fig. 1B), which suggests HPP-c3-s1 is a homogeneous polysaccharide. The PAGE results supported the HPGPC analysis. 

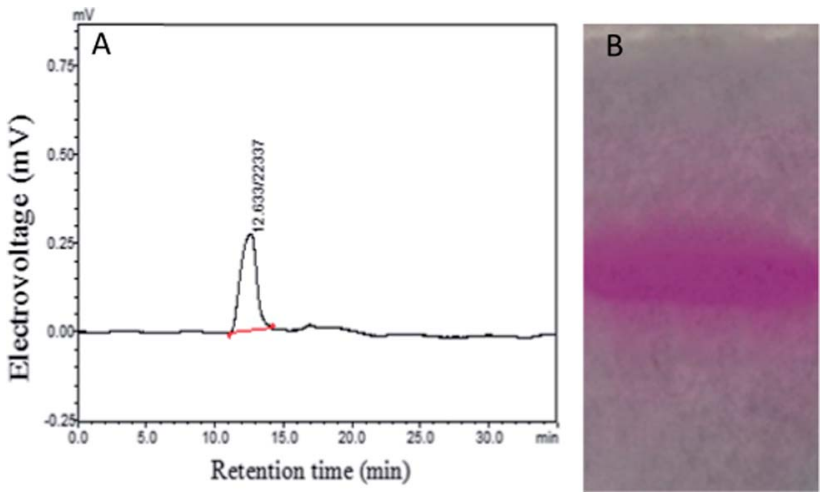

Fig. 1 The homogeneity and molecular weight of HPP-c3-s1 were investigated using HPGPC (A) and PAGE (B). The spectrum of HPP-c3s1 was determined through a LC-10A HPLC system equipped with a RID-10A detector. Ultra-pure water was used as the mobile phase at a flow rate of $0.6 \mathrm{~mL} \mathrm{~min}{ }^{-1}$. The column temperature was $35^{\circ} \mathrm{C}$ and the injection volume was $20 \mu \mathrm{L}$. Electropherogram of HPP-c3-s1, stained according to the periodic acid-Schiffs reagent (PAS) method. The separating and stacking gel solutions were $8 \%$ and $3.5 \%$, respectively. Electrophoresis of samples was initially at $40 \mathrm{~V}$ for $20 \mathrm{~min}$ and then at $110 \mathrm{~V}$ for $1 \mathrm{~h}$. The gels were rinsed, oxidized, rinsed, stained, destained, and scanned according to Material and methods 2.3.

\subsection{Periodate oxidation and smith degradation analysis}

Upon periodate oxidation, HPP-c3-s1 produced $0.0028 \mathrm{mmol}$ formic acid, indicating the likely presence of $1 \rightarrow 6$ glycosyl linkage(s). Based on the sodium periodate calibration curve, $0.0287 \mathrm{mmol}$ of sodium periodate was consumed during oxidation. The consumption of sodium periodate being more than twice the amount of formic acid produced indicates there may be numerous glycosyl linkages, such as $1 \rightarrow 2$ and $1 \rightarrow 4$, because these two linkages consume sodium periodate, but produce no formic acid. ${ }^{25}$

After Smith degradation of the periodate-oxidized product, the degradation products were examined by GC-MS (Fig. 2). Ribose, glucose, and mannose were present, indicating the presence of corresponding linkages that could not be oxidized, namely $1 \rightarrow 3$ linkages. In addition, glycerol was found in the degradation products, suggesting the presence of glycosidic linkages, such as $1 \rightarrow 2$ or $1 \rightarrow 6$. Erythritol was also present, revealing HPP-c3-s1 may contain a $1 \rightarrow 4$ linkage. Therefore, the results from periodate oxidation-Smith degradation suggest that HPP-c3-s1 contains $1 \rightarrow 2,1 \rightarrow 3,1 \rightarrow 4$, and small number of $1 \rightarrow 6$ linkages.

\subsection{FTIR spectroscopy}

The FTIR spectrum of HPP-c3-s1 contained bands characteristics of polysaccharides with absorbance modes from 400 to $4000 \mathrm{~cm}^{-1}$ (Fig. 3). The intense and broad band at $3396.25 \mathrm{~cm}^{-1}$ was from the stretching vibration of an $\mathrm{O}-\mathrm{H}$, suggesting the presence of intra- and intermolecular hydrogen bonds. The absorption peaks at 2933.97 and 1200 to $1400 \mathrm{~cm}^{-1}$ correspond to the stretching and angular vibration of a C-H bond, indicating HPP-c3-s1 is a carbohydrate. ${ }^{26}$ The moderate strength absorption peak around $1649.97 \mathrm{~cm}^{-1}$ was from $\mathrm{C}=\mathrm{O}$ stretching vibration, suggesting that $\mathrm{HPP}-\mathrm{c} 3-\mathrm{s} 1$

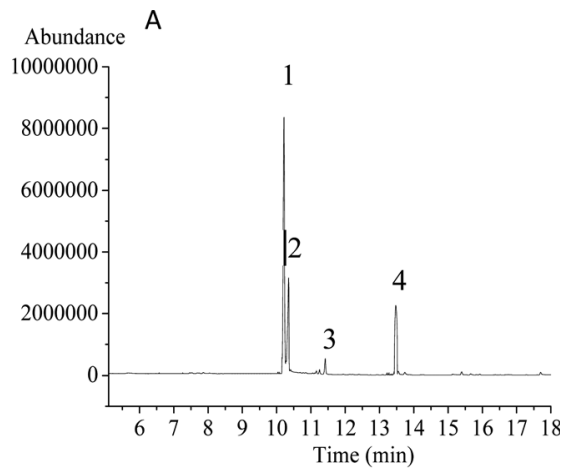

B

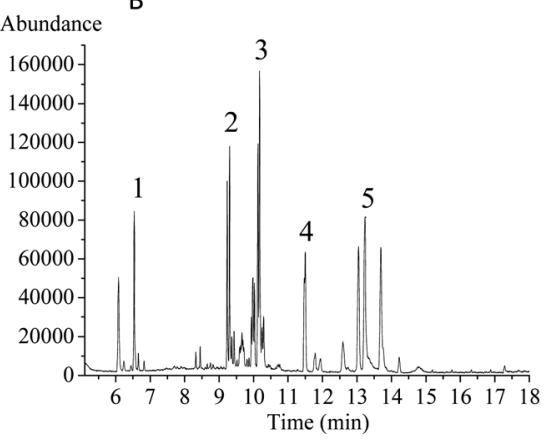

Fig. 2 GC-MS profiles of derivatives of monosaccharide standards (A) and Smith degradation products of HPP-c3-s1 (B). The monosaccharide standards and samples were treated according to Material and methods 2.4. The GC-MS conditions were as follows: carrier gas, high purity helium; flow rate, $1.2 \mathrm{~mL} \mathrm{~min}^{-1}$; El ion source; electron energy, $70 \mathrm{eV}$; transmission line temperature, $280{ }^{\circ} \mathrm{C}$. (A) Four monosaccharide standards. (1) Ribose (Rib); (2) arabinose (Ara); (3) mannose (Man); (4) glucose (Glc); (B) HPP-c3-s1. (1) Glycerol; (2) erythritol; (3) ribose (Rib); (4) mannose (Man); (5) glucose (Glc).

may contain acetyl or uronic acid groups. The absorption peak at $1411.06 \mathrm{~cm}^{-1}$ likely relates to $\mathrm{C}-\mathrm{H}$ bending vibration, while the band at $1239.00 \mathrm{~cm}^{-1}$ can be assigned to the stretching of an $\mathrm{S}=\mathrm{O}$ bond. The strong absorption peak at $1039.39 \mathrm{~cm}^{-1}$ is from C-O stretching in a primary alcohol $\mathrm{OH}$, which is the characteristic absorption peak of the pyranose carbohydrate ring. ${ }^{27}$ These results indicate that HPP-c3-s1 is a sulphated pyranose.

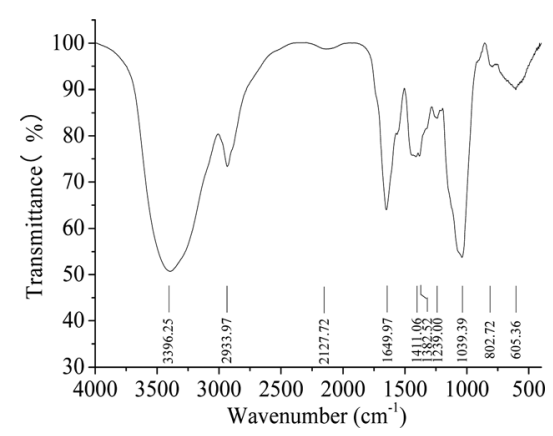

Fig. 3 Fourier transform infrared spectroscopy analysis of the HPPc3-s1 fraction. Sample, $1 \mathrm{mg}$, mixed with potassium bromide $(100 \mathrm{mg}$ ) was analyzed on a Nexus FTIR with a DTGS $\mathrm{KBr}$ detector. The absorptive spectra were recorded in the frequency range of 4000$400 \mathrm{~cm}^{-1}$. 


\subsection{Analysis of ${ }^{\mathbf{1}} \mathrm{H}$ NMR spectroscopy}

To verify the characteristic chemical shifts and corresponding functional groups of HPP-c3-s1, ${ }^{1} \mathrm{H}$ NMR spectroscopy was used (Fig. 4). There were anomeric hydrogen signals between $\delta 3.0$ and $5.0 \mathrm{ppm}$. The chemical shifts of $\mathrm{C} 1$ protons at less than and more than $5.0 \mathrm{ppm}$ can be attributed to then $\beta$ - and $\alpha$-anomeric configurations, respectively, confirming both $\alpha$ and $\beta$ configurations may be present in HPP-c3-s1. ${ }^{28}$ TPC3-1 molecule from green tea (Camellia sinensis L.) also has the anomeric carbon signs of both $\alpha$ and $\beta$ configurations. ${ }^{29}$ The residual hydrogen of $\mathrm{D}_{2} \mathrm{O}$ was observed at $4.70 \mathrm{ppm}$ and the signal at $4.01 \mathrm{ppm}$ was related to the hydrogen next to the functional $-\mathrm{OH}$ group. A group of overlapping signals between $\delta 3.3$ and $4.0 \mathrm{ppm}$ correspond to C2-C6 protons, which was difficult to analyze. Spectra at around $\delta 1.93$ and $2.12 \mathrm{ppm}$ suggest the presence of acetyl groups. The signals between $\delta 0.99$ and $3.66 \mathrm{ppm}$ indicate an amino group. ${ }^{30}$ No characteristic chemical shift was observed near $\delta 5.4 \mathrm{ppm}$, indicating HPP-c3-s1 is a pyranose. Due to the absence of ${ }^{13} \mathrm{C}$ NMR data, linkage data on the sugar chains is unclear.

\subsection{Atomic force microscopy of HPP-c3-s1}

Fig. 5 presents the nanostructural properties of HPP-c3-s1, where two different concentrations, 5 and $50 \mu \mathrm{g} \mathrm{mL}{ }^{-1}$, of the material were used for AFM. At a concentration of $50 \mu \mathrm{g} \mathrm{mL}{ }^{-1}$, HPP-c3-s1 formed different sized small aggregates with an average diameter of 80-150 nm (Fig. 5A). Spontaneous aggregates with spherical and looped structures, as well as linear and branched forms, formed at the $5 \mu \mathrm{g} \mathrm{mL} \mathrm{mL}^{-1}$ concentration (Fig. 5B). Fig. 5C (5 $\mu \mathrm{g}$ $\mathrm{mL}^{-1}$ ) clearly presents that HPP-c3-s1 contained both linear and branched structures, where the width of the linear molecules ranged from 23 to $36 \mathrm{~nm}$ and the height from about 0.6 to $1.5 \mathrm{~nm}$. According to periodate oxidation, Smith degradation analysis, and FTIR spectroscopy, HPP-c3-s1 formed through branched chains. This corresponded with the small aggregates and linear and branched structures observed on AFM. In recent years, some reports described the use of AFM to determine the molecular structure of polysaccharides. CP2-c2-s2 from Cordyceps militaris showed different aggregates and networks from $5 \mu \mathrm{g}$ $\mathrm{mL}^{-1}$ to $250 \mu \mathrm{g} \mathrm{mL}{ }^{-1}$. At low concentrations $\left(5 \mu \mathrm{g} \mathrm{mL}{ }^{-1}\right)$, the

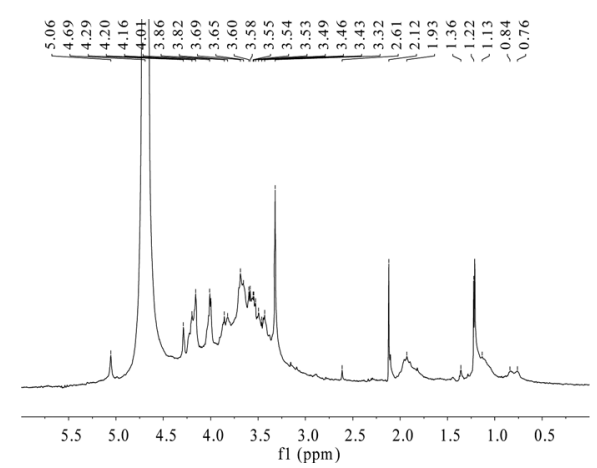

Fig. $4{ }^{1} \mathrm{H}$ NMR spectra of HPP-c3-s1. The spectra were recorded using an Avance II NMR spectrometer at $600 \mathrm{MHz}$ in $\mathrm{D}_{2} \mathrm{O}$. Freezedried sample, $10 \mathrm{mg}$, was exchanged three times with $\mathrm{D}_{2} \mathrm{O}$ and spectra were measured in the range of $0-6 \mathrm{ppm}$.

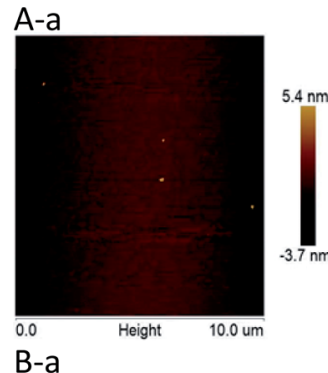

$A-b$

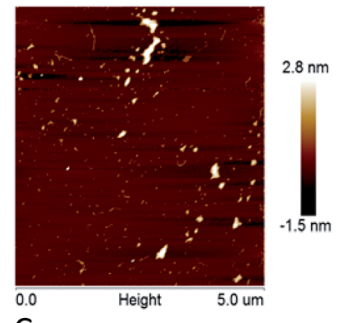

B-b

C-a

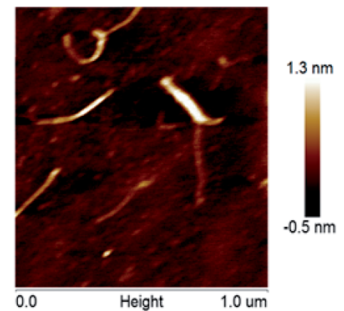

C-b

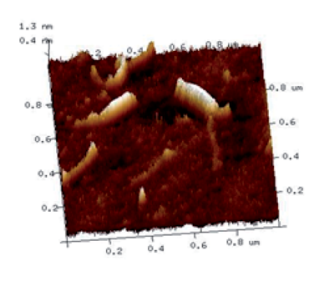

Fig. 5 AFM images of HPP-c3-s1 at concentrations of (A) $50 \mu \mathrm{g} \mathrm{mL}^{-1}$, (B) $5 \mu \mathrm{g} \mathrm{mL}$, and (C) $5 \mu \mathrm{g} \mathrm{mL} \mathrm{m}^{-1}$. (a) Height mode image; (b) 3D visualization. Exactly $5 \mu \mathrm{L}$ of each sample was dropped onto freshly cleaved mica and air-dried overnight. AFM imaging was recorded on MultiMode 8 instrument at room temperature of about $22-25^{\circ} \mathrm{C}$ and a relative humidity of $50-60 \%$ on mica sheet. The tape of the tip was Silicon Tip on Nitride Lever and the cantilever was RTEST (T: $650 \mathrm{~nm}$, f0: $70 \mathrm{kHz}, \mathrm{k}: 0.4 \mathrm{~N} \mathrm{~m}^{-1}$ ).

CP2-c2-s2 molecules formed small dispersed aggregates with different sizes. At $50 \mu \mathrm{g} \mathrm{mL}{ }^{-1}$ clearly formed rod-like helical network structures. ${ }^{31}$ AMBG from Mesona blumes gum showed spherical lumps at $1 \mu \mathrm{g} \mathrm{mL}{ }^{-1}$ but an irregular worm-like shape at $10 \mu \mathrm{g} \mathrm{mL}{ }^{-1}$. $^{2}$

Determining the polysaccharide chemical structure and analysis of the carbohydrate chain were very difficult. At present, most studies on polysaccharide structure concentrate on the primary structure. Because polysaccharides have a large relative molecular weight, the sugar residues are often connected with methyl, acetyl, sulfate, and phosphate groups, rendering the primary structure of polysaccharides complex and difficult to analyse. Nevertheless, structural analysis plays an important role in the study of polysaccharides. It is also the basis of the relationship between the molecular structure and biological functions of polysaccharides. At present, there are many methods to analyze polysaccharide structure and typical methods, including chemical analysis, such as acid hydrolysis method, periodate oxidation, and Smith degradation, and instrumental analysis, such as infrared spectroscopy, NMR, and GC/MS. ${ }^{33}$ In this study, chemical analysis in the forms of periodate oxidation and Smith degradation, as well as instrumental 

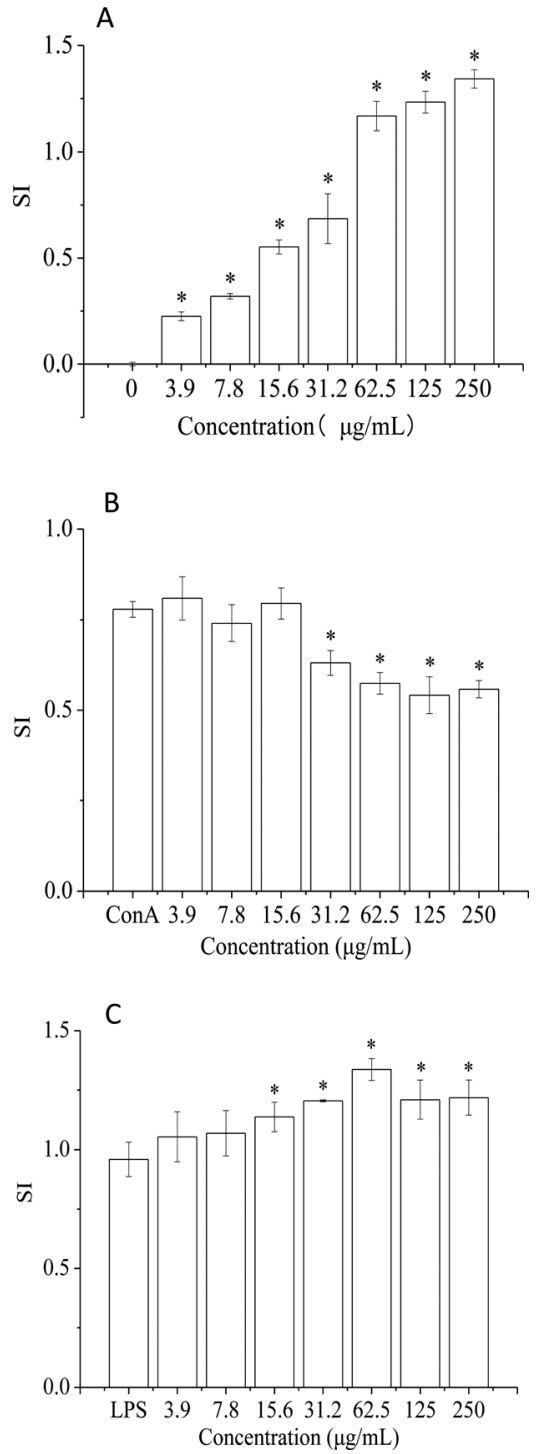

Fig. 6 Effects of HPP-c3-s1 on mouse splenocyte lymphocyte simulation: (A) polysaccharide stimulation alone, (B) ConA-induced T lymphocytes, and (C) LPS-induced B lymphocytes. After $44 \mathrm{~h}$, the cell viability was measured by MTT assay. Each value was presented as mean $\pm \mathrm{SD},(n=6) . * p<0.05$ compared to positive control.

analysis through HPGPC, IR, NMR, GC/MS, and AFM, were used to determine the primary structure of HPP-c3-s1. The primary structure of polysaccharide HPP-c3-s1 is composed of four monosaccharides, including D-ribose, D-arabinose, $\mathrm{D}$ mannose, and D-glucose with $1 \rightarrow 2,1 \rightarrow 3,1 \rightarrow 4$, and trace amounts of $1 \rightarrow 6$ glycosidic linkages. HPP-c3-s1 is a pyranose containing an amino and an O-acetyl group. The sugar chains contain two glycosidic configurations, $\alpha$ and $\beta$, and are primarily in the $\beta$ configuration. It was observed the HPPc3-s1 nanostructure contains linear and branched forms at a concentration of $5 \mu \mathrm{g} \mathrm{mL} L^{-1}$, and different sized aggregates at a higher concentration of $50 \mu \mathrm{g} \mathrm{mL} \mathrm{m}^{-1}$. This important information aids in revealing the primary and advanced structures of the polysaccharide isolated from $\mathrm{H}$. pluvialis.

\subsection{Effect of HPP-c3-s1 on spleen lymphocyte proliferation}

The immune system has a crucial role in health and consists of tissues, cells, and molecules that protect organisms from infections. ${ }^{34}$ In recent years, alga polysaccharides have been shown to modulate the human immune system and protect human health..$^{35}$ In this study, the effect of HPP-c3-s1 on splenic lymphocytes was investigated to characterize its mechanism of the immunoregulatory activity. As shown in Fig. 6, treatment with HPP-c3-s1 significantly increased splenocyte and B lymphocyte proliferation. However, HPP-c3-s1 had no effect on the proliferative activity of ConA-induced $\mathrm{T}$ lymphocytes at doses of 3.9 to $250 \mu \mathrm{g} \mathrm{mL}^{-1}$ compared to the ConA alone group. Even at the doses of 31.2 to $250 \mu \mathrm{g} \mathrm{mL}^{-1}$, HPP-c3-s1 had significantly decrease the proliferative activity of ConA-induced $\mathrm{T}$ lymphocytes. The possible reason was that the high concentration of polysaccharide and ConA caused overstimulation of cells under the synergistic effect, thus inhibiting the proliferation of cells. The specific reasons are still to be studied further. At doses of 15.6 to $250 \mu \mathrm{g} \mathrm{mL} \mathrm{mL}^{-1}$, HPP-c3-s1 significantly promoted splenocyte and LPS-stimulated splenocyte proliferation, indicating that HPP-c3-s1 is directly mitogenic for B lymphocytes. The SI (Stimulus Index) of $62.5 \mu \mathrm{g} \mathrm{mL} \mathrm{m}^{-1} \mathrm{HPP}-\mathrm{c} 3-\mathrm{s} 1$ in LPS-induced B lymphocytes was 1.34 (Fig. 6C). However, at doses of HPP-c3-s1 higher than $62.5 \mu \mathrm{g} \mathrm{mL}^{-1}$, B lymphocyte proliferation gradually decreased. These results indicate that HPP-c3-s1 has remarkable immunostimulatory activity, where HPP-c3-s1 promotion of lymphocyte proliferation is mainly through LPS-induced B lymphocyte proliferation.

The immune system prevents diseases through biological processes. Diseases of the immune system can lead to autoimmunity, inflammation, and even cancer. The spleen is an important peripheral immune organ and is where immune cells perform a number of their immune-related functions. Splenic cells consist of several types of immune cells, including $\mathrm{T}$ and $\mathrm{B}$ lymphocytes, which can directly respond to the immune regulation of the organism. ${ }^{36} \mathrm{~T}$ and $\mathrm{B}$ lymphocytes are two important types of immunologically active cells. The former is mainly responsible for cellular immunity, while the latter are the only cells that produce antibodies and are the primary humoral immune cells. ${ }^{37}$ ConA and LPS are mitogenic agents for T cells

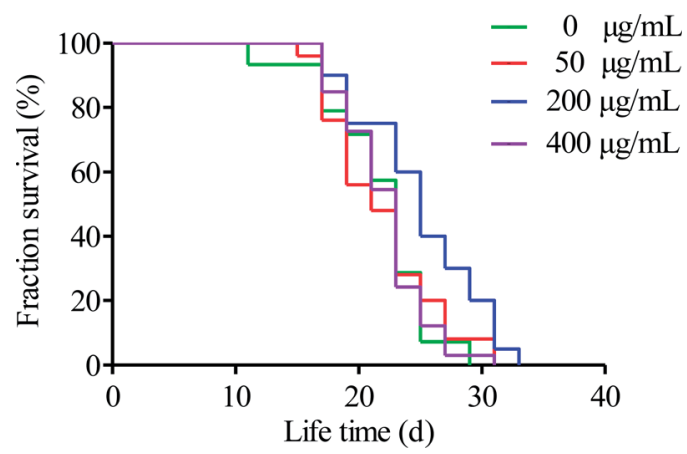

Fig. 7 Effects of HPP-c3-s1 at concentrations of 50, 200, and $400 \mu \mathrm{g}$ $\mathrm{mL}^{-1}$ on lifespan extension of $C$. elegans. Day 0 refers to the first day that $C$. elegans ( $L 4$ stage) were transferred to NGM plates. Worms were scored daily for survival. Three independent trials were performed. 

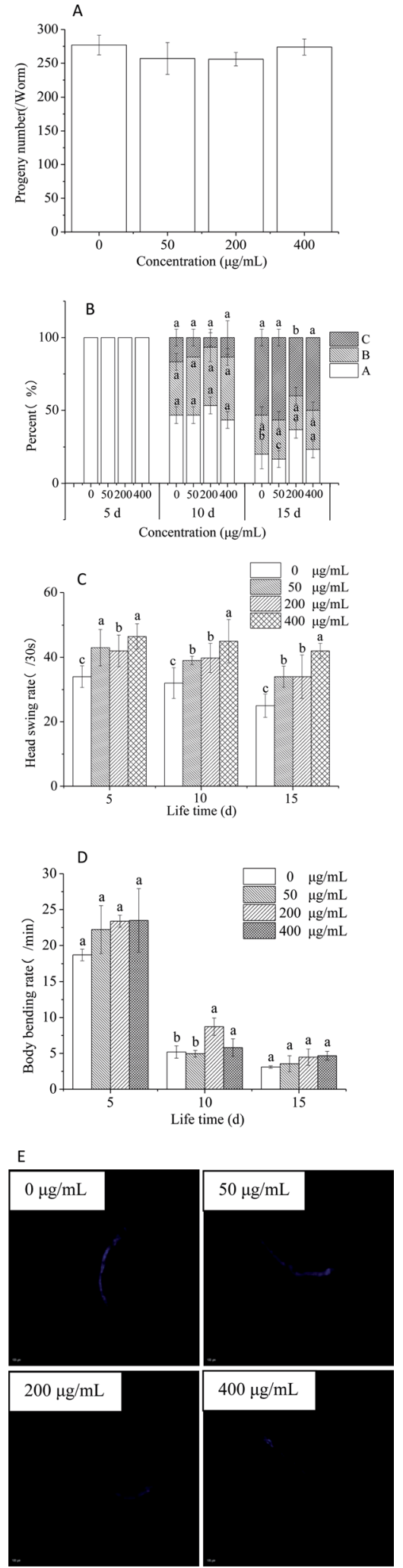

Fig. 8 Effects of different concentrations of HPP-c3-s1 on age-related changes in physiologic parameters of $C$. elegans. The fecundity (A), body movement $(B)$, head swing rate $(C)$, and body bending rates $(D)$ were counted under a dissecting microscope. (E) The intestinal lipofuscin fluorescence of day 8 worms was photographed using a fluorescence microscope. The lipofuscin level was quantified using ImageJ software by determining the average pixel intensity in each worm. The experiment was and B cells, respectively. It is generally accepted that drugs capable of enhancing ConA- or LPS-promotion of spleen cell proliferation act through promotion of proliferation of $\mathrm{T}$ or $\mathrm{B}$ cells. Studies have shown that a polysaccharide from Spirulina not only stimulated the proliferation of lymphocytes and induced the secretion of IL-2 in mouse spleen cells, but also promoted transformation of these cells in vitro following induction by ConA in a dose-dependent manner. ${ }^{38}$ Treatment of mice immunocompromised with cyclophosphamide treatment with Spirulina polysaccharide displayed a significant promotion in proliferation of spleen cells and thymocytes. Spirulina polysaccharide significantly enhanced the transformation of mouse spleen lymphocytes induced by ConA, thus enhancing the immune activity of immune cells. ${ }^{39}$ In addition, a highmolecular-weight polysaccharide extract from the cyanobacterium Arthrospira platensis enhanced human adaptive immune responses. ${ }^{40}$ A new low molecular weight sulfated polysaccharide GLP-2 isolated from Gracilaria lemaneiformis significantly improved the proliferation and pinocytosis of RAW264.7 cells and promoted the production of reactive oxygen species, nitric oxide, interleukin-6, and tumor necrosis factor-alpha by activating expression of inducible nitric oxide synthesis, interleukin-6, and tumor necrosis factor-alpha. ${ }^{41}$ In this paper, the proliferation of $\mathrm{T}$ and $\mathrm{B}$ lymphocytes was studied in terms of enhancement of immune activity by HPP-c3-s1. It was found that HPP-c3-s1 not only independently stimulated spleen cell proliferation, but certain concentrations also significantly promoted mouse spleen B lymphocyte LPS-induced proliferation in a dose-dependent manner. These results show that HPPc3-s1 binds to the B lymphocyte receptor and plays a role similar to that of LPS mitogen, thus stimulating B lymphocyte proliferation. However, no such activity was observed for T lymphocytes. In addition, the effect of high concentrations of polysaccharide on cell proliferation under synergistic action inhibited proliferation, although the mechanism behind this remains to be delineated.

\subsection{Anti-aging activity}

C. elegans can quickly perceive external environmental factors, such as light, heat, heavy metals, and poisons. It is an excellent model organism that is very sensitive to the environment. At present, it is widely used in the field of medicine and basic biological research..$^{42}$ Up to now, there are few studies on the anti-aging activity of microalgae polysaccharides using C. elegans model. Polysaccharides from Chinese traditional herbal medicines, including Cordyceps militaris, ${ }^{31}$ Bletilla striata ${ }^{43}$ and Panax notoginseng, ${ }^{44}$ have been shown to extend the lifespan of C. elegans. When evaluating nematode aging, lifespan is the main index used. ${ }^{45}$

To evaluate the effect of HPP-c3-s1 on the lifespan of C. elegans, $\mathrm{N} 2$ wild type nematodes were treated with concentrations

repeated three times and images were captured using a $20 \times$ objective. Data are expressed as the mean $\pm \mathrm{SD},(n=3)$. The data were statistically analyzed using one way-ANOVA (SPSS 17.0). Differences between the data were considered significant at $p<0.05$. 
of 50, 200, and $400 \mu \mathrm{g} \mathrm{mL} \mathrm{m}^{-1}$. Treatment with $200 \mu \mathrm{g} \mathrm{mL} \mathrm{m}^{-1} \mathrm{HPP}-$ c3-s1 extended the mean survival of the worms, while 50 and $400 \mu \mathrm{g} \mathrm{mL} \mathrm{m}^{-1}$ had no obvious effect compared to the control group (Fig. 7). It was observed that $200 \mu \mathrm{g} \mathrm{mL}{ }^{-1}$ HPP-c3-s1 significantly increased the average lifespan of the nematodes by $14.92 \%(p<0.05)$ and the maximum life increased by about $3.33 \mathrm{~d}$. While 50 and $400 \mu \mathrm{g} \mathrm{mL}{ }^{-1}$ HPP-c3-s1 had no significant effect on the mean lifespan of worms $(p>0.05)$, they did increase the maximum lifespan of the worms.

Several physiologic parameters, including fecundity, body movement, rates of head swings and body bending, and intestinal lipofuscinosis, were also observed to evaluate the antiaging activity. ${ }^{46}$ None of the treatment groups displayed significant variation in progeny production. Thus, HPP-c3-s1 had no impact on reproduction in C. elegans (Fig. 8A). Motility classes were determined by scoring the locomotion phenotype of worms by gently prodding them with a platinum wire. 'A' worms move spontaneously away from the touch; ' $\mathrm{B}$ ' worms did respond to touch, but with somewhat uncoordinated and move slowly; ' $\mathrm{C}$ ' worms were unable to move, but they were clearly alive. On day 5 , all the groups of worms could move spontaneously without touching a stimulus, i.e. displayed movement. On day 10 , the proportion of nematodes in state $\mathrm{A}$ of each group began to decrease, while those in state B significantly increased (Fig. 8B). In addition, the number of nematodes in state $\mathrm{A}$ in the $200 \mu \mathrm{g} \mathrm{mL}{ }^{-1}$ HPP-c3-s1 was higher than in the other three groups and exhibited significant difference compared with the control group on day $15(p<0.05)$. These results demonstrate HPP-c3-s1 slows down deterioration of motility in the worms. We observed a decline in the rate of head swing on days 5, 10, and 15 for both the treated (HPP-c3-s1) and untreated N2 worms, however the rate in the treated group remained significantly higher than the control group $(p<0.05)$. Moreover, the rate of head swing in the worms treated with $400 \mu \mathrm{g} \mathrm{mL}^{-1} \mathrm{HPP}$ c3-s1 was much higher than in the other three groups, suggesting that HPP-c3-s1 plays a significant role in delaying the rate of head swing in nematodes (Fig. 8C). The mean body bending rate decreased within 15 days (Fig. 8D). Compared to the control group, the mean body bending rate slightly increased in the HPP-c3-s1-treated group on days 5 and 15. However, treatment with $200 \mu \mathrm{g} \mathrm{mL}{ }^{-1}$ HPP-c3-s1 significantly increased the rate of body bending $(p<0.05)$. Intracellular lipofuscin level is a prominent marker for aging and age-related cellular damage. ${ }^{47}$ To measure intestinal lipofuscin accumulation, ImageJ software was used to calculate levels in worms on day 8 (Fig. 8E). It was found $200 \mu \mathrm{g} \mathrm{mL} \mathrm{mL}^{-1} \mathrm{HPP}-\mathrm{c} 3-\mathrm{s} 1$ significantly reduced lipofuscin fluorescence compared to the control group $(p<0.05)$. Therefore, HPP-c3-s1 partly decreased the accumulation of intestinal lipofuscin and, thus, extended the lifespan of nematode.

\section{Conclusions}

In summary, the aqueous polysaccharide HPP-c3-s1 was isolated from the microalgae $H$. pluvialis and determined to have a molecular weight of $23413 \mathrm{kDa}$ in this study. HPP-c3-s1 was characterized as a pyranose containing $1 \rightarrow 2,1 \rightarrow 4,1 \rightarrow 3$, and probably a few $1 \rightarrow 6$ glycosyl linkages, an amino group, and an O-acetyl group The sugar chain contains both $\alpha$ and $\beta$ glycosidic configurations, but primarily consists of the $\beta$ configuration. Furthermore, when HPP-c3-s1 is at a concentration of $5 \mu \mathrm{g} \mathrm{mL}^{-1}$, both linear and branched structures exist, where the width of the linear molecules ranges from 23 to $36 \mathrm{~nm}$ and the height from about $0.6 \mathrm{~nm}$ to $1.5 \mathrm{~nm}$. By contrast, when HPP-c3-s1 was at a concentration of $50 \mu \mathrm{g} \mathrm{mL}{ }^{-1}$, small aggregates of different sizes with an average diameter of 80$150 \mathrm{~nm}$ formed. In addition, HPP-c3-s1 exhibited immunostimulatory effects on splenocyte lymphocytes, particularly $\mathrm{B}$ lymphocytes. In anti-aging assays using C. elegans, HPP-c3-s1 extended the mean survival without adversely impact reproduction. Furthermore, HPP-c3-s1 could alleviate the decline in physiological function caused by senility in C. elegans, such as body movement, rates of head swings and body bending, and accumulation of intestinal lipofuscinosis. Therefore, HPP-c3-s1 displayed remarkable immunomodulatory and anti-aging activity, indicating that HPP-c3-s1 has the potential to be used as a novel functional food.

\section{Conflicts of interest}

There are no conflicts to declare.

\section{Acknowledgements}

This study was financially supported by the Guangzhou Science and Technology Plan Project (Grant No. 201707010035), the Guangdong Natural Science Foundation of China (Grant No. 2017A030313150), and the National Natural Science Foundation of China (Grant No. 31401481).

\section{References}

1 (a) L. Song, X. L. Chen, X. D. Liu, F. B. Zhang, L. F. Hu, Y. Yue, K. C. Li and P. C. Li, Characterization and comparison of the structural features, immune-modulatory and anti-avian influenza virus activities conferred by three algal sulfated polysaccharides, Mar. Drugs, 2016, 14(1), 4; (b) A. Ekelhof and M. Melkonian, Microalgal cultivation in porous substrate bioreactor for extracellular polysaccharide production, J. Appl. Phys., 2017, 29(3), 1115-1122; (c) E. Hussain, L. J. Wang, B. Jiang, S. Riaz, G. Y. Butt and D. Y. Shi, A review of the components of brown seaweeds as potential candidates in cancer therapy, RSC Adv., 2016, 6(15), 12592-12610.

2 (a) D. H. Ngo and S. K. Kim, Sulfated polysaccharides as bioactive agents from marine algae, Int. J. Biol. Macromol., 2013, 62(11), 70; (b) T. H. Silva, A. Alves, E. G. Popa, L. L. Reys, M. E. Gomes, R. A. Sousa, S. S. Silva, J. F. Mano and R. L. Reis, Marine algae sulfated polysaccharides for tissue engineering and drug delivery approaches, Biomatter, 2012, 2(4), 278; (c) A. C. M. Leodido, L. E. C. Costa, T. S. L. Araujo, D. S. Costa, N. A. Sousa, L. K. M. Souza, F. B. M. Sousa, M. D. S. Filho, D. F. P. Vasconcelos and F. R. P. Silva, Anti-diarrhoeal 
therapeutic potential and safety assessment of sulphated polysaccharide fraction from Gracilaria intermedia seaweed in mice, Int. J. Biol. Macromol., 2017, 97, 34-45.

3 C. Faggio, M. Pagano, A. Dottore, G. Genovese and M. Morabito, Evaluation of anticoagulant activity of two algal polysaccharides, Nat. Prod. Res., 2016, 30(17), 19341937.

4 I. P. S. Fernando, J. W. Nah and Y. J. Jeon, Potential antiinflammatory natural products from marine algae, Environ. Toxicol. Pharmacol., 2016, 48, 22-30.

5 E. Hussain, L. J. Wang, B. Jiang, S. Riaz, G. Y. Butt and D. Y. Shi, A review of the components of brown seaweeds as potential candidates in cancer therapy, $R S C A d v$., 2016, 6(15), 12592-12610.

6 M. Takei, T. Kuda, M. Eda, A. Shikano, H. Takahashi and B. Kimura, Antioxidant and fermentation properties of aqueous solutions of dried algal products from the Boso Peninsula, Japan, Food Biosci., 2017, 19, 85-91.

7 H. W. Chen, T. S. Yang, M. J. Chen, Y. C. Chang, E. I. C. Wang, C. L. Ho, Y. J. Lai, C. C. Yu, J. C. Chou and L. K. P. Chao, Purification and immunomodulating activity of C-phycocyanin from Spirulina platensis cultured using power plant flue gas, Process Biochem., 2014, 49(8), 13371344.

8 (a) J. Matos, C. Cardoso, N. M. Bandarra and C. Afonso, Microalgae as healthy ingredients for functional food: a review, Food Funct., 2017, 8(8), 2672-2685; (b) C. E. C. C. Ejike, S. A. Collins, N. Balasuriya, A. K. Swanson, B. Mason and C. C. Udenigwe, Prospects of microalgae proteins in producing peptide-based functional foods for promoting cardiovascular health, Trends Food Sci. Technol., 2017, 59, 30-36.

9 (a) J. Qi and S. M. Kim, Characterization and immunomodulatory activities of polysaccharides extracted from green alga Chlorella ellipsoidea, Int. J. Biol. Macromol., 2017, 95, 106-114; (b) Y. Sun, H. Wang, G. Guo, Y. Pu and B. Yan, The isolation and antioxidant activity of polysaccharides from the marine microalgae Isochrysis galbana, Carbohydr. Polym., 2014, 113, 22-31; (c) L. Sun, L. Wang and Y. Zhou, Immunomodulation and antitumor activities of different-molecular-weight polysaccharides from Porphyridium cruentum, Carbohydr. Polym., 2012, 87(2), 1206-1210; (d) S. Santoyo, M. Plaza, L. Jaime, E. Ibañez, G. Reglero and F. G. Señorans, Pressurized liquid extraction as an alternative process to obtain antiviral agents from the edible microalga Chlorella vulgaris, J. Agric. Food Chem., 2010, 58(15), 8522.

10 J. K. Park, Z. H. Kim and C. G. Lee, Characterization and immunostimulating activity of a water-soluble polysaccharide isolated from Haematococcus lacustris, Biotechnol. Bioprocess Eng., 2011, 16(6), 1090-1098.

11 C. K. Lee, H. S. Kim, R. N. Ju, J. R. Nam, M. J. Lee, J. H. Yim, H. K. Lee and E. De Clercq, Anti-picornavirus activity and other antiviral activity of sulfated exopolysaccharide from the marine microalga Gyrodinium impudicum strain KG03, Antiviral Research, 2009, 82(2), A40.
12 G. Panis and J. R. Carreon, Commercial astaxanthin production derived by green alga Haematococcus pluvialis: A microalgae process model and a techno-economic assessment all through production line, Algal Res., 2016, 18, 175-190.

13 (a) Y. Liu, S. H. Wang and Y. Liu, Physicochemical properties and antioxidant activity of polysaccharide from Haematococcus pluvialis extracted by three different methods, Food Sci. (Beijing), 2015, 36(6), 161-168; (b) Y. M. Feng, Extraction, isolation, structural characterization and anticoagulation of polysaccharides from four kinds of green seaweeds, J. Ocean Univ. China, 2012.

$14 \mathrm{M}$. Zhang, X. J. Liu and X. Liu, Optimization of process in extracting polysaccharide assisted by ultrasonic wave from Haematococcus pluvialis residues, Food Sci. Technol. Int., 2016, 37(20), 254-258.

15 X. Liu, Y. Huang, Y. Chen and Y. Cao, Partial structural characterization, as well as immunomodulatory and antiaging activities of CP2-c2-s2 polysaccharide from Cordyceps militaris, RSC Adv., 2016, 6, 104094-104103.

16 D. Luo, X. Yuan, Y. Zeng, K. Nie, Z. Li and Z. Wang, Structure elucidation of a major fucopyranose-rich heteropolysaccharide (STP-II) from Sargassum thunbergii, Carbohydr. Polym., 2016, 143, 1-8.

17 Y. Y. Ren, Z. Y. Zhu, H. Q. Sun and L. J. Chen, Structural characterization and inhibition on alpha-glucosidase activity of acidic polysaccharide from Annona squamosal, Carbohydr. Polym., 2017, 174, 1-12.

18 Y. Han, J. Wu, T. Liu, Y. Hu, Q. Zheng, B. Wang, H. Lin and $\mathrm{X}$. Li, Separation, characterization and anticancer activities of a sulfated polysaccharide from Undaria pinnatifida, Int. J. Biol. Macromol., 2015, 83(3), 42-49.

19 A. Mishra, K. Kavita and B. Jha, Characterization of extracellular polymeric substances produced by microalgae Dunaliella salina, Carbohydr. Polym., 2011, 83(2), 852-857.

20 D. Tang, P. Attar, A. Q. Li and E. H. Dowell, Complex dynamics of a pyranose ring structure molecule attached to an atomic force microscope, Nonlinear Dyn., 2006, 45(34), 283-303.

21 X. Chen, W. Nie, S. Fan, J. Zhang, Y. Wang, J. Lu and L. Jin, A polysaccharide from Sargassum fusiforme protects against immunosuppression in cyclophosphamide-treated mice, Carbohydr. Polym., 2012, 90(2), 1114-1119.

22 A. Pant, S. K. Saikia, V. Shukla, J. Asthana, B. A. Akhoon and R. Pandey, Beta-caryophyllene modulates expression of stress response genes and mediates longevity in Caenorhabditis elegans, Nonlinear Dyn., 2014, 57(9), 81-95.

23 E. M. Vayndorf, S. S. Lee and R. H. Liu, Whole apple extracts increase lifespan, healthspan and resistance to stress in Caenorhabditis elegans, J. Funct. Foods, 2013, 5(3), 1235-1243.

24 X. Liu, Q. Luo, Y. Cao, T. Goulette, X. Liu and H. Xiao, Mechanism of different stereoisomeric astaxanthin in resistance to oxidative stress in Caenorhabditis elegans, $J$. Food Sci., 2016, 81(9), H2280-H2287. 
25 T. Feng, Z. B. Gu and Z. Y. Jin, Structural studies of an acidic polysaccharide of Mesona blumes gum, J. Sci. Food Agric., 2008, 88(1), 24-34.

26 L. Wang, H. M. Liu and G. Y. Qin, Structure characterization and antioxidant activity of polysaccharides from Chinese quince seed meal, Food Chem., 2017, 234, 314-322.

27 W. Liu, Y. M. Liu, R. Zhu, P. Yu, W. S. Lu, C. Pan, W. B. Yao and X. D. Gao, Structure characterization, chemical and enzymatic degradation, and chain conformation of an acidic polysaccharide from Lycium barbarum L., Carbohydr. Polym., 2016, 147, 114-124.

28 Y. J. Gong, J. Zhang, F. Gao, J. W. Zhou, Z. N. Xiang, C. G. Zhou, L. S. Wan and J. C. Chen, Structure features and in vitro hypoglycemic activities of polysaccharides from different species of Maidong, Carbohydr. Polym., 2017, 173, 215-222.

29 H. X. Chen, Z. S. Wang, X. M. Lu and B. J. Xie, Isolation and chemical characterization of a polysaccharide from green tea (Camellia sinensis L.), J. Sci. Food Agric., 2008, 88(14), 25232528.

30 M. I. Pereira, A. C. Ruthes, E. R. Carbonero, R. Marcon, C. H. Baggio, C. S. Freitas, A. R. S. Santos, S. Eliasaro, G. L. Sassaki and P. A. J. Gorin, Chemical structure and selected biological properties of a glucomannan from the lichenized fungus Heterodermia obscurata, Phytochemistry, 2010, 71(17-18), 2132-2139.

31 X. Liu, Y. Huang, Y. Chen and Y. Cao, Partial structural characterization, as well as immunomodulatory and antiaging activities of CP2-c2-s2 polysaccharide from Cordyceps militaris, RSC Adv., 2016, (6), 104094-104103.

32 T. Feng, Z. B. Gu and Z. Y. Jin, Structural studies of an acidic polysaccharide of Mesona blumes gum, J. Sci. Food Agric., 2008, 88(1), 24-34.

33 (a) X. L. Li, B. Zhang, J. Li, J. Zhou, X. L. He, L. Ye, J. Zou, C. G. Wu, X. P. Zhang and W. H. Peng, Purification, characterization, and complement fixation activity of acidic polysaccharides from Tuber sinoaestivum, LWT-Food Sci. Technol., 2017, 85, 82-88; (b) R. B. Kolsi, J. Fakhfakh, F. Krichen, I. Jribi, A. Chiarore, F. P. Patti, C. Blecker, N. Allouche, H. Belghith and K. Belghith, Structural characterization and functional properties of antihypertensive Cymodocea nodosa sulfated polysaccharide, Carbohydr. Polym., 2016, 151, 511-522.

34 L. V. Hooper, D. R. Littman and A. J. Macpherson, Interactions between the microbiota and the immune system, Science, 2012, 336(6086), 1268-1273.

35 R. Pangestuti and S. K. Kim, Biological activities and health benefit effects of natural pigments derived from marine algae, J. Funct. Foods, 2011, 3(4), 255-266.

36 (a) Z. Y. Zhu, M. Meng, H. Sun, Y. Li, Y. Y. Ren and Y. Zhang, Immunostimulatory activity of glycopeptides from Paecilomyces sinensis under normal and cyclophosphamide induced immunosuppressive conditions in mice models, Food Funct., 2016, 7(8), 3566-3576; (b) W. T. Fan, P. M. Zheng, Y. Wang, P. Hao, J. Z. Liu and X. N. Zhao,
Analysis of immunostimulatory activity of polysaccharide extracted from Yu-Ping-Feng in vitro and in vivo, Biomed. Pharmacother., 2017, 93, 146-155.

37 T. R. Mosmann and R. L. Coffman, TH1 and TH2 cells: different patterns of lymphokine secretion lead to different functional properties, Annu. Rev. Immunol., 2003, 7(7), 145.

$38 \mathrm{X}$. Wang and $\mathrm{H}$. Yu, Effect of spirulina on immunologic function of mice in vitro experiment, J. Tradit. Chin. Med., 2004, 20(9), 482-483.

$39 \mathrm{~W}$. Chen, S. Lv and D. He, The effect of spirulina platensis polysaccharide on lyphocyte proliferation and IFN- $\gamma$ induction in immunosuppressive model mice, Lishizhen Medicine and Materia Medica Research, 2008, 19(4), 941-942.

40 M. Lobner, A. Walsted, R. Larsen, K. Bendtzen and C. H. Nielsen, Enhancement of human adaptive immune responses by administration of a high-molecular-weight polysaccharide extract from the cyanobacterium Arthrospira platensis, J. Med. Food, 2008, 11(2), 313-322.

41 Y. L. Ren, G. Q. Zheng, L. J. You, L. R. Wen, C. Li, X. Fu and L. Zhou, Structural characterization and macrophage immunomodulatory activity of a polysaccharide isolated from Gracilaria lemaneiformis, J. Funct. Foods, 2017, 33, 286-296.

42 (a) P. I. Racz, M. Wildwater, M. Rooseboom, E. Kerkhof, R. Pieters, E. S. Yebra-Pimentel, R. P. Dirks, H. P. Spaink, C. Smulders and G. F. Whale, Application of Caenorhabditis elegans (nematode) and Danio rerio embryo (zebrafish) as model systems to screen for. developmental and reproductive toxicity of Piperazine compounds, Toxicol. in Vitro, 2017, 44, 11-16; (b) T. Kaletta and M. O. Hengartner, Finding function in novel targets: C. elegans as a model organism, Nat. Rev. Drug Discovery, 2006, 5, 387-399.

43 Y. S. Zhang, T. Lv, M. Li, T. Xue, H. Liu, W. M. Zhang, X. Y. Ding and Z. H. Zhuang, Anti-aging effect of polysaccharide from Bletilla striata on nematode Caenorhabditis elegans, Pharmacogn. Mag., 2015, 11(43), 449-454.

44 S. Feng, H. Cheng, Z. Xu, S. Shen, M. Yuan, J. Liu and C. Ding, Thermal stress resistance and aging effects of Panax notoginseng polysaccharides on Caenorhabditis elegans, Int. J. Biol. Macromol., 2015, 81, 188-194.

45 X. Zhao, L. Lu, Y. Qi, M. Li and L. Zhou, Emodin extends lifespan of Caenorhabditis elegans through insulin/IGF-1 signaling pathway depending on DAF-16 and SIR-2.1, Biosci., Biotechnol., Biochem., 2017, 81(10), 1908-1916.

46 Y. Chen, B. Onken, H. Chen, S. Xiao, X. Liu, M. Driscoll, Y. Cao and Q. Huang, Mechanism of longevity extension of Caenorhabditis elegans induced by pentagalloyl glucose isolated from eucalyptus leaves, J. Agric. Food Chem., 2014, 62(15), 3422-3431.

47 J. Lee, G. Kwon and Y. H. Lim, Elucidating the mechanism of weissella-dependent lifespan extension in Caenorhabditis elegans, Sci. Rep., 2015, 5, 17128. 